九州大学学術情報リポジトリ

Kyushu University Institutional Repository

\title{
SUSPENSION DESIGN OF A SEMI-SUBMERSIBLE PLATFORM
}

\section{Zhu, Hongzhong}

Research and Education Center for Advanced Energy Materials, Devices, and Systems, Kyushu University

$\mathrm{Hu}$, Changhong

Research Institute for Applied Mechanics, Kyushu University

Liu, Yingyi

Research Institute for Applied Mechanics, Kyushu University

Liao, Kangping

College of Ship Building Engineering, Harbin Engineering University

http://hdl. handle. net/2324/4752553

出版情報: Proceedings of the ASME 2016 35th International Conference on Ocean, Offshore and Arctic Engineering. 7, 2016-10-18. The American Society of Mechanical Engineers : ASME バージョン:

権利関係: Copyright（c）2016 by ASME 
Proceedings of Proceedings of the ASME 2016 35th International Conference on Ocean,
Offshore and Arctic Engineering
OMAE2016
June 19-24, 2016, Busan, Korea

OMAE2016-54347

\section{SUSPENSION DESIGN OF A SEMI-SUBMERSIBLE PLATFORM}

\author{
Hongzhong Zhu* \\ Research and Education Center for \\ Advanced Energy Materials, Devices, \\ and Systems, Kyushu University \\ Kasuga 816-8580, Japan \\ Email: zhuhongzhong@riam.kyushu-u.ac.jp
}

\author{
Changhong Hu \\ Yingyi Liu \\ Research Institute for Applied \\ Mechanics, Kyushu University \\ Kasuga 816-8580, \\ Japan
}

\author{
Kangping Liao \\ College of Ship Building Engineering, \\ Harbin Engineering University \\ Nangang District, Harbin 150001, \\ China
}

\begin{abstract}
With the development of ocean energy exploration, reliable and low cost semi-submersible platforms are expected to develop. The maximum pitching amplitude of a floater for floating offshore wind turbine should be less than a few degrees to avoid fatigue failure. In this paper, a novel conceptual design of a new type semi-submersible with suspensions for suppressing the pitch motion is presented. Many wave energy dissipation devices, such as add-on wave energy converters to a floating platform, could be regarded as the suspension system in our design. Firstly, linear models are applied to approximate the radiation forces and wave exciting forces so that the whole motion system is represented by a state-space model. Then, we show that design of suspensions leads to synthesize a controller via solving a constrained $\mathbf{H}_{\infty}$ optimization problem. Finally, numerical examples are performed to verify the design and it can be shown that the pitch motion of the semi-submersible platform is remarkably reduced.
\end{abstract}

\section{INTRODUCTION}

A semi-submersible platform is a specialised marine vessel used in a number of offshore applications such as offshore drilling rigs and heavy lift cranes. As the development of offshore wind energy exploration, reliable and low cost semisubmersible platforms are increasingly required [1]. Although the platforms are designed with good sea-keeping performance and good stability, environmental forces induced by wind, waves and ocean currents can, however, induce undesired heave\&pitch motion, which would remarkably reduce the fatigue life of devices on the platforms [2-4]. Suzuki and Sato (2007) have performed some pioneering work on investigating the effects of motion of floating platform on the strength of offshore wind turbine blades, and they come to the result that pitching with amplitude of 5 degrees will lead to a $50 \%$ increase of sectional modulus of a blade to avoid fatigue failure [5].

In the last decade there have been some floating platforms built for full scale experimentation like GustosMSC Tri-Floater [6], WindFloat [7], and the V-shape semi-submersible floater [8]. Most of them are designed with three or four rigidly-connected columns having a small waterplane area to decrease the effects of waves. Even by sophisticated analysis and design, the maximum inclination of the floaters would also reach to 10 degrees according to model tests [6]. In order to further reduce the motions, various methods and state-of-art structures were proposed in the literature. In $[9,10]$, novel water-entrapment plates with large horizontal skirts were designed to increase the added-mass and viscous loads to semi-submersible platform. The natural periods of the platform in heave, pitch and roll can be adjusted to avoid the resonance with the environmental forces. Though the water-entrapment plates can be systematically designed considering strength analysis and fatigue loads, the fabrication cost, however, would limit its applications. In addition, Roddier and Cermelli (2013) have proposed an interesting concept of structure consisting of three column tubes which are partly filled with water [11]. The water can be pumped between the three columns 
to balance out the environment forces so that the inclination of platform could be controlled. A major advantage of the concept is that the pitch motion can be controlled by pumping the water from some columns to the others. However, the response may be too slow to wind turbulence and non-regular waves.

Recent studies also focus on combining wave energy converters (WEC) with floating offshore wind turbine (FOWT) for a better utilization of marine space and lower installation cost $[12,13]$. Though there are many advantages, the combination also introduces the inter-influence between WEC and FOWT that makes the whole system complex. Perez and Iglesias (2012) reported that particular attention should be paid to the wind turbine foundation affected by the installation of the WEC since the inclination of foundation may exceed the design limit [14]. Hence, optimal design of the combination system becomes an urgent work.

In this paper, a conceptual design of semi-submersible with suspensions is presented. The system consists of a platform and some vertical columns which are connected to the platform via spring and damper. The columns can also be regarded as the WEC in the combined wave/wind energy system in which the dampers are considered as the power take-off devices. The purpose of this study is to minimize the pitch motion of the platform by designing the coefficients of spring and damper under some given wave state. Though the concept is similar to the suspension design of land-based vehicles, the nonlinear hydrodynamic interaction arises between different submerged components of the system in the wave propagation process, which makes the design of suspensions much more complicated than the latter.

The remainder of this paper is organized as follows. Firstly, the conceptual design of a semi-submersible platform is introduced. Secondly, the mathematical models of the platform and the wave exciting forces are presented. Then, a method to optimally design the parameters of spring and damper is proposed, and some numerical examples are given to demonstrate the effectiveness of the design.

\section{Semi-Submersible Platform and Problem Description}

The schematic of conceptual design is shown in Fig. 1. As the first step of the research, a two-dimensional model which is bilaterally symmetric is considered for simplicity. The platform is supported both by the pontoon via the pillars and by the vertical columns located at the both ends via spring and damper. In the figure, $z_{1}$ is the displacement of column $1, z_{2}$ the displacement of column $2, \theta$ the pitch angle from their equilibrium positions. $z_{p_{1}}$ and $z_{p_{2}}$ are the displacemente of the ends of platform in vertical direction. The columns can be regarded as the WEC in which the dampers are considered as the power take-off devices. Note that the spring and damper can offer more flexibility to design the semi-submersible in the presence of waves and winds. Some experimental investigations of using WECs in

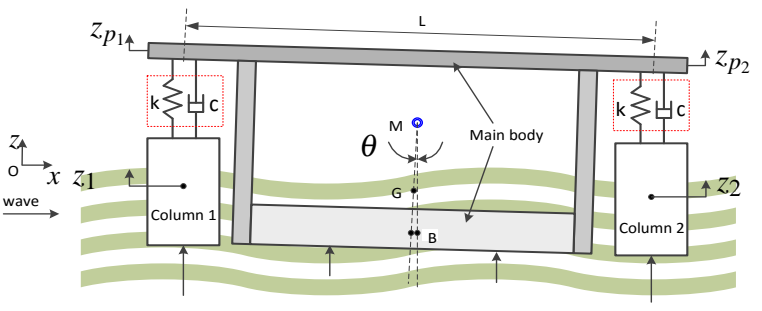

FIGURE 1. Schematic of conceptual design.

a semi-submersible have been discussed in [12].

Compared to wave forces, the variation of wind forces is relatively low and long term. Hence we focus on reducing the wave induced motions, especially the pitch motion, by optimally designing the coefficients of spring and damper. In the following, we also assume that the diameter of the columns is much smaller than wave length so that the heave motion of columns is only taken into account.

\section{Mathematical Model of Semi-Submersible}

Assuming that the motion amplitudes of each component are small, we can express the motion equations as

$$
\begin{aligned}
J_{p} \ddot{\theta} & =\tau_{\mathrm{rad}}+\tau_{\mathrm{res}}+\tau_{\mathrm{exc}}+\frac{L}{2}\left(f_{1}-f_{2}\right) \\
m_{c} \ddot{z}_{1} & =F_{\mathrm{rad}_{1}}+F_{\mathrm{res}_{1}}+F_{\mathrm{exc}_{1}}-f_{1} \\
m_{c} \ddot{z}_{2} & =F_{\mathrm{rad}_{2}}+F_{\mathrm{res}_{2}}+F_{\mathrm{exc}_{2}}-f_{2}
\end{aligned}
$$

where $J_{p}$ is the inertia moment of the main body to the center of rotation, $m_{c}$ is the mass of one column, $\tau_{\mathrm{rad}}, F_{\mathrm{rad}_{1}}, F_{\mathrm{rad}_{2}}$ are corresponding to the radiation forces arising from the change in momentum of the fluid due to the motion of the system, $\tau_{\text {res }}, F_{\text {res }_{1}}, F_{\text {res }_{2}}$ are restoring forces due to the gravity and buoyancy, and $\tau_{\mathrm{exc}}, F_{\mathrm{exc}_{1}}, F_{\mathrm{exc}_{2}}$ represent the wave excitation forces consisting of Froude-Krylov forces and diffraction forces. The terms $f_{1}, f_{2}$ are the counteracting forces from the spring-damper sets that

$$
\begin{aligned}
& f_{1}=k z_{r_{1}}+c \dot{z}_{r_{1}}, \\
& f_{2}=k z_{r_{2}}+c \dot{z}_{r_{2}} .
\end{aligned}
$$

Here, $z_{r_{1}} \doteq z_{1}-z_{p_{1}}, z_{r_{2}} \doteq z_{2}-z_{p_{2}}$ are the relative positions of the platform and the columns, the terms $k$ and $c$ are the coefficients of the spring and the damper, respectively.

The radiation force of pitch motion can be expressed by [15]

$$
\tau_{\mathrm{rad}}=-J_{\infty} \ddot{\theta}-\int_{0}^{t} K_{\theta_{r}}(t-\tau) \dot{\theta}(\tau) d \tau
$$


In the right-side of equation, the first term owes to the acceleration of the platform and $J_{\infty}$ is a constant positive added inertia moment. The second term represents fluid-memory effects that capture the energy transfer from the motion of platform to the radiated waves and $K_{\theta_{r}}(t)$ is the impulse response function of the radiation in pitch motion. The convolution integral term could be approximated by following linear subsystem

$$
\left\{\begin{array}{l}
\dot{x}_{\theta_{r}}(t)=A_{\theta_{r}} x_{\theta_{r}}(t)+B_{\theta_{r}} \dot{\theta}(t) \\
y_{\theta_{r}}(t)=C_{\theta_{r}} x_{\theta_{r}}(t) \approx \int_{0}^{t} K_{\theta_{r}}(t-\tau) \dot{\theta}(\tau) d \tau
\end{array}\right.
$$

where $x_{\theta_{r}} \in \mathbf{R}^{n \times 1}$ is state vector, and $A_{\theta_{r}}, B_{\theta_{r}}, C_{\theta_{r}}$ are system matrices which can be obtained from theory or experiments by system identification [16].

By combining the equations (1), (6) and (7), and applying the linearized restoring force

$$
\tau_{\mathrm{res}}=-\rho g V \overline{\mathrm{GM}} \theta
$$

where $\rho$ is the density of water, $g$ the gravitational acceleration, $V$ the displaced volume of water and $\overline{\mathrm{GM}}$ the meta-centric height, the pitch motion equation of the main body (1) becomes

$$
\mathscr{G}_{\theta}:\left\{\begin{array}{l}
\dot{x}_{\theta}(t)=A_{\theta} x_{\theta}(t)+B_{\theta_{a}} \tau_{\mathrm{exc}}(t)+B_{\theta_{b}} u_{\theta_{b}}(t) \\
y_{\theta}(t)=C_{\theta} x_{\theta}(t)
\end{array}\right.
$$

Here, $y_{\theta}=[\theta, \dot{\theta}]^{T}$ is output vector, $x_{\theta}=\left[x_{\theta_{r}}^{T}, \theta, \dot{\theta}\right]^{T}$ is state vector, $u_{\theta_{b}}=\left[f_{1}, f_{2}\right]^{T}$ is force vector from (4) (5), and the matrices are

$$
\begin{gathered}
A_{\theta}=\left[\begin{array}{ccc}
A_{\theta_{r}} & 0_{n \times 1} & B_{\theta_{r}} \\
0_{1 \times n} & 0 & 1 \\
-\frac{C_{\theta_{r}}}{J_{p}+J_{\infty}} & -\frac{\rho g V \overline{\mathrm{GM}}}{J_{p}+J_{\infty}} & 0
\end{array}\right], B_{\theta_{a}}=\left[\begin{array}{c}
0_{n \times 1} \\
0 \\
\frac{1}{J_{p}+J_{\infty}}
\end{array}\right], \\
B_{\theta_{b}}=\left[\begin{array}{cc}
0_{n \times 1} & 0_{n \times 1} \\
0 & 0 \\
\frac{L}{2} \frac{1}{J_{p}+J_{\infty}} & -\frac{L}{2} \frac{1}{J_{p}+J_{\infty}}
\end{array}\right], C_{\theta}=\left[\begin{array}{lll}
0_{1 \times n} & 1 & 0 \\
0_{1 \times n} & 0 & 1
\end{array}\right],
\end{gathered}
$$

where $0_{i \times j}$ is the zero matrix with $i$ rows and $j$ columns.

Similarly, the heave motion equations of the columns at the sides of the platform become

$$
\begin{aligned}
& \mathscr{G}_{c 1}:\left\{\begin{array}{l}
\dot{x}_{c 1}(t)=A_{c} x_{c 1}(t)+B_{c_{a}} F_{\mathrm{exc}_{1}}(t)+B_{c_{b}} f_{1}(t) \\
y_{c 1}(t)=C_{c} x_{c 1}(t),
\end{array}\right. \\
& \mathscr{G}_{c 2}:\left\{\begin{array}{l}
\dot{x}_{c 2}(t)=A_{c} x_{c 2}(t)+B_{c_{a}} F_{\mathrm{exc}_{2}}(t)+B_{c_{b}} f_{2}(t) \\
y_{c 2}(t)=C_{c} x_{c 2}(t)
\end{array}\right.
\end{aligned}
$$

where $x_{c 1}=\left[x_{c 1_{r}}^{T}, z_{1}, \dot{z}_{1}\right]^{T}$ and $x_{c 2}=\left[x_{c 2_{r}}^{T}, z_{2}, \dot{z}_{2}\right]^{T}$ are state vectors, $y_{c 1}=\left[z_{1}, \dot{z}_{1}\right]^{T}$ and $y_{c 2}=\left[z_{2}, \dot{z}_{2}\right]^{T}$ are output vectors. The vectors $x_{c 1_{r}}$ and $x_{c 2_{r}}$ in $x_{c 1}$ and $x_{c 2}$ are the state vectors of subsystems to approximate the radiation forces in heave direction. The matrices $A_{c}, B_{c_{a}}, B_{c_{b}}, C_{c}$ can be derived by the same approach as (9).

\section{Mathematical Model of Wave Exciting Forces}

According to linear theory, the excitation force is proportional to the incident wave elevation that

$$
f_{e}(t)=\int_{-\infty}^{\infty} h_{f}(t-\tau) \eta(0, \tau) d \tau
$$

Here, $\eta(x, t)$ is the incident wave elevation where $x$ is the horizontal coordinate in the direction of wave propagation, and $\eta(0, t)$ is the wave elevation at the origin. The term $h_{f}(t)$ is the impulse response function of excitation force coefficient which can be calculated by potential theory. For simplicity, we assume the wave is in X-direction, as shown in Fig. 1, and the origin of wave is at the vertical axis through the centre of the Column 1. Then we use a linear model to approximate the excitation force $F_{\mathrm{exc}_{1}}$ on the Column 1 expressed by

$$
\mathscr{F}_{e_{1}}(s)=\frac{b_{c_{0}} s^{r}+b_{c_{1}} s^{r-1}+\cdots+b_{c_{r-1}} s+b_{c_{r}}}{s^{r}+a_{c_{1}} s^{r-1}+\cdots+a_{c_{r-1}} s+a_{c_{r}}} \cdot \pi(0, s),
$$

where $s$ is the Laplace operator, $\mathscr{F}_{e 1}$ are the Laplace transform of $F_{\mathrm{exc}_{1}}$. The term $\pi(0, s)$ is the Laplace transform of the wave $\eta(0, t)$. The coefficients $b_{c_{0}}, b_{c_{1}}, \cdots, b_{c_{r}}$ and $a_{c_{0}}, a_{c_{1}}, \cdots, a_{c_{r}}$ are the variables to be tuned to fit the real system via system identification, see [17] for comprehensive overviews. Similarly, the excitation forces $\tau_{\mathrm{exc}}$, and $F_{\mathrm{exc}_{2}}$ can be modelled as

$$
\begin{aligned}
& \mathscr{T}_{e}(s)=\frac{b_{\tau_{0}} s^{r}+b_{\tau_{1}} s^{r-1}+\cdots+b_{\tau_{r-1}} s+b_{\tau_{r}}}{s^{r}+a_{\tau_{1}} s^{r-1}+\cdots+a_{\tau_{r-1}} s+a_{\tau_{r}}} \cdot \pi\left(\frac{L}{2}, s\right), \\
& \mathscr{F}_{e_{2}}(s)=\frac{b_{c_{0}} s^{r}+b_{c_{1}} s^{r-1}+\cdots+b_{c_{r-1}} s+b_{c_{r}}}{s^{r}+a_{c_{1}} s^{r-1}+\cdots+a_{c_{r-1}} s+a_{c_{r}}} \cdot \pi(L, s),
\end{aligned}
$$

where $\pi(d, s)\left(d=\frac{L}{2}\right.$ or $\left.L\right)$ is the Laplace transform of the wave $\eta(d, t) . \pi(d, s)$ can be approximated as $\pi(d, s) \approx e^{-T_{d} s} \pi(0, s)$ that $T_{d}$ is wave propagation period from the origin $x=0$ to a distance $d$ at $x=d$. The first order Padé-approximation can be exploited for linearize this delayed term. In the case of irregular incident wave, the dominant wave frequency should be applied to 


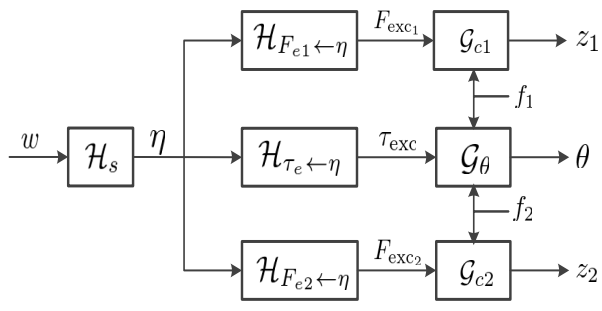

FIGURE 2. Block diagram of the whole model.

calculate these periods. Then, the realization of models (13-15) can be expressed as

$$
\begin{aligned}
& \mathscr{H}_{F_{e_{1}} \leftarrow \eta}: \frac{\mathscr{F}_{e_{1}}(s)}{\pi(s)}=\left[\begin{array}{c|c}
A_{F_{e_{1}} \eta} & B_{F_{e_{1}} \eta} \\
\hline C_{F_{e_{1}}} \eta & D_{F_{e_{1}}}
\end{array}\right], \\
& \mathscr{H}_{T_{e} \leftarrow \eta}: \frac{\mathscr{T}_{e}(s)}{\pi(s)}=\left[\begin{array}{c|c|c}
A_{T_{e}} \eta & B_{T_{e} \eta} \\
\hline C_{T_{e} \eta} & D_{T_{e}}
\end{array}\right], \\
& \mathscr{H}_{F_{e_{2}} \leftarrow \eta}: \frac{\mathscr{F}_{e_{2}}(s)}{\pi(s)}=\left[\begin{array}{c|c|c}
A_{F_{e_{2}}} \eta & B_{F_{e_{2}} \eta} \\
\hline C_{F_{e_{2}}} \eta & D_{F_{e_{2}}} \eta
\end{array}\right],
\end{aligned}
$$

where the notation

$$
\left[\begin{array}{l|l}
A & B \\
\hline C & D
\end{array}\right]:=C(s I-A)^{-1} B+D
$$

is used. The matrices in (16-18) could be calculated by the coefficients in (13-15), respectively.

Fig. 2 shows the integration of the models given by (9-11) and (16-18). In the figure, in order to apply linear control theories for synthesis, a linear approximation of wave spectral density function, which is denoted as $\mathscr{H}_{s}$, is also introduced. The signal $w$ in the figure is a zero-mean Gaussian white noise process with unit power. In this study, a widely used second-order system is employed to approximate the wave spectral density function [18]:

$$
\mathscr{H}_{s}(s)=\frac{2 \lambda \omega_{0} \sigma s}{s^{2}+2 \lambda \omega_{0} s+\omega_{0}^{2}}
$$

Here, $\lambda$ is a damping coefficient, $\sigma$ is a constant describing the wave intensity and $\omega_{0}$ is the dominating wave frequency. A corresponding linear state-space model is

$$
\begin{aligned}
& \dot{x}_{w}(t)=A_{w} x_{w}(t)+B_{w} w(t) \\
& \eta(0, t)=C_{w} x_{w}(t)
\end{aligned}
$$

where $x_{w}$ is the state vector. The matrices are expressed by

$$
\begin{aligned}
& A_{w}=\left[\begin{array}{ll}
0 & 1 \\
-\omega_{0}^{2} & -2 \lambda \omega_{0}
\end{array}\right], B_{w}=\left[\begin{array}{l}
0 \\
2 \lambda \omega_{0} \sigma
\end{array}\right] \\
& C_{w}=\left[\begin{array}{ll}
0 & 1
\end{array}\right]
\end{aligned}
$$

In the next section, we will show that the optimal design of spring and damper leads to a feedback controller design problem.

\section{Design}

Integrating together the models shown in Fig. 2, we can obtain a system described by

$$
\begin{aligned}
& \dot{x}(t)=A x(t)+B_{1} w(t)+B_{2} u(t), \\
& p(t)=C_{1} x(t) \\
& y(t)=C_{2} x(t)
\end{aligned}
$$

where $x=\left[x_{c 1}^{T}, x_{a}^{T}, x_{\theta}^{T}, x_{b}^{T}, x_{c 2}^{T}, x_{c}^{T}, x_{w}^{T}\right]^{T}$ is the state vector, $p=\theta$ is performance output signal to be regulated, $y=$ $\left[z_{r_{1}}, \dot{z}_{r_{1}}, z_{r_{2}}, \dot{z}_{r_{2}}\right]^{T}$ is the output vector that the elements depict the relative motions. The vectors $x_{a}, x_{b}$ and $x_{c}$ are the state vectors of subsystems (16), (17) and (18), respectively. The inputs are the zero-mean Gaussian white noise process $w$ and the force vector $u=\left[f_{1}, f_{2}\right]^{T}$ from the spring-damper sets. The matrices are given in (23) where $p=n+r$.

According to (4) and (5), we can obtain that

$$
\left[\begin{array}{l}
f_{1} \\
f_{2}
\end{array}\right]=K y,
$$

where

$$
K=\left[\begin{array}{llll}
k & c & 0 & 0 \\
0 & 0 & k & c
\end{array}\right]
$$

Obviously, the design of spring-damper set equals to determine the output feedback controller $K$ for the system (22) to minimize the effects of input $w$. The relationship between the inputs and the outputs is shown in Fig. 3. Denoting the transfer matrix from the input $w$ to the performance output $\theta$ as $\mathscr{H}_{\theta w}$, we can obtain that

$$
\mathscr{H}_{\theta w}(s)=C_{1}(s I-\tilde{A})^{-1} B_{1}
$$

where $I$ is identity matrix with appropriate dimension and $\tilde{A}=$ $A+B_{2} K C_{2}$. The design problem leads to the following minimize 


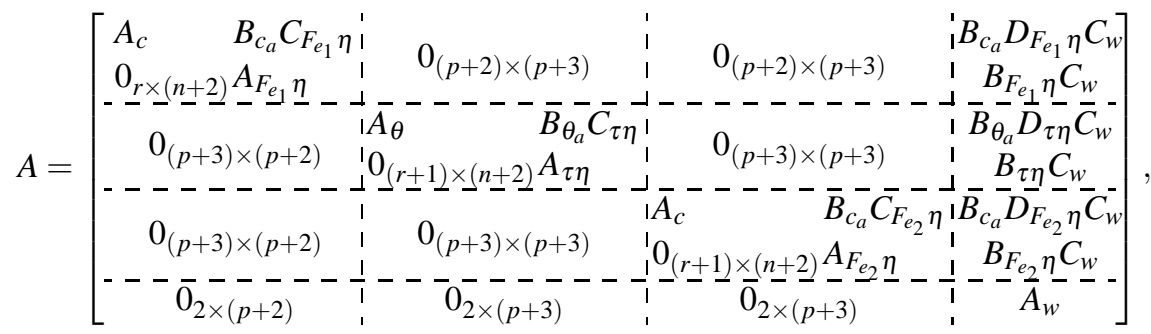

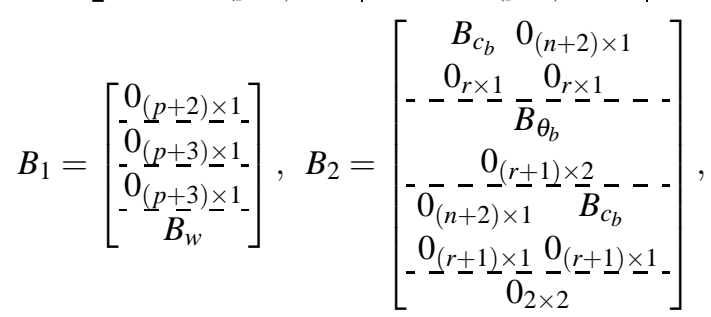

$$
\begin{aligned}
& C_{1}=\left[\begin{array}{lllll}
0_{1 \times(p+2)},{ }^{\prime} 0_{1 \times n} & 1 & 0 & 0_{1 \times(r+1)}{ }^{\prime} 0_{1 \times(p+3)}{ }^{1}, 0_{1 \times 2}
\end{array}\right], \\
& C_{2}=\left[\begin{array}{c:cccc}
C_{c} & 0_{2 \times r}-\frac{L}{2} C_{\theta} & 0_{2 \times(r+1)} & 0_{2 \times(p+3)} & 0_{2 \times 2} \\
0_{2 \times(p+2)} & \frac{L}{2} C_{\theta} & 0_{2 \times(r+1)} & C_{c} 0_{2 \times(r+1)} & 0_{2 \times 2}
\end{array}\right],
\end{aligned}
$$

problem:

$$
\min _{k, c} \quad\left\|\mathscr{H}_{\theta w}\right\|_{\infty}
$$

subject to: Condition (25)

$$
\begin{aligned}
& k_{l} \leq k \leq k_{h} \\
& c_{l} \leq c \leq c_{h},
\end{aligned}
$$

where $\left[k_{l}, k_{h}\right]$ and $\left[c_{l}, c_{h}\right]$ are the assigned proper ranges for $k$ and $c$ from the point of view of safety and robustness. Owing to the constraint conditions, the optimization problem (27) cannot be analytically solved, for instance, by LMI approach. Therefore, the following numerical approach is proposed considering ranges of sea wave frequency instead of the whole frequency domain that:

$$
\begin{aligned}
\min _{k, c} \max _{i=1}^{m} & \left\|C_{1}\left(j \omega_{i} I-\tilde{A}\right)^{-1} B_{1}\right\| \\
\text { subject to: } & \text { Condition }(25) \\
& k_{l} \leq k \leq k_{h} \\
& c_{l} \leq c \leq c_{h}
\end{aligned}
$$

where $\omega_{i}(i=1,2, \cdots, m)$ are the typical wave frequencies exploited for design. The optimization problem (28) can be solved by several nonlinear programming solvers, such as Genetic Algorithm and Particle Swarm Optimization method [19].

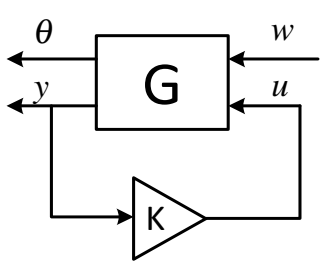

FIGURE 3. Design of spring-damper systems is equal to determine the feedback controller $K$.

\section{Numerical Example}

As an example, let us consider a conceptual model shown in Fig. 1. The radius and the mass of columns are $50 \mathrm{~mm}$ and $1 \mathrm{~kg}$. The horizontal pontoon is a cylinder with radius $50 \mathrm{~mm}$ and length $600 \mathrm{~mm}$, and sealed by two vertical pillars with thickness $10 \mathrm{~mm}$ at the both sides. The width of the pillars is set as same as the diameter of pontoon. The mass of main body is $5.385 \mathrm{~kg}$, and its center of gravity is located $30 \mathrm{~mm}$ above the keel line. The draft of the whole body is $170 \mathrm{~mm}$. Besides, the inertia of moment of the main part to the rotation center is $0.62 \mathrm{~kg} \cdot \mathrm{m}^{2}$. Assume the semi-submersible in this study is applied for non-fully developed sea with large depth, the modified Pierson-Moskowitz (MPM) Spectrum is exploited to describe the sea state:

$$
S(\omega)=A \omega^{-5} \exp \left(-B \omega^{-4}\right),
$$




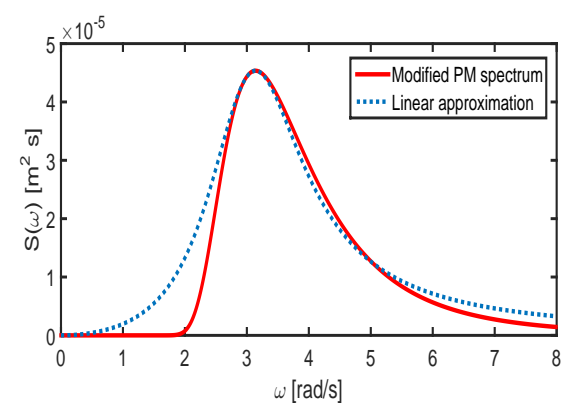

FIGURE 4. Plot with solid line showing the modified PiersonMoskowitz spectra for $H_{s}=0.01 \mathrm{~m}$ and $T_{z}=1.4207 \mathrm{~s}$. The linear approximation by (20) is plotted with dotted line.

where $A$ and $B$ are parameters assigned by $A=\frac{4 \pi^{3} H_{s}^{2}}{T_{z}^{4}}, B=\frac{16 \pi^{3}}{T_{z}^{4}}$ ( $H_{s}$ is the significant wave height and $T_{z}$ is the average zerocrossing wave period). In this study, we set $H_{s}=0.01 \mathrm{~m}$ and $T_{z}=1.4207 \mathrm{~s}$ as wave conditions and the dominating wave frequency is $\omega_{0}=3.14 \mathrm{rad} / \mathrm{s}$. The wave spectra is shown in Fig. 4 . The linear approximation by transfer function (20) is also plotted in the figure by using curve fitting method. The resulting wave intensity is $\sigma=0.0067$ and damping coefficient is $\lambda=0.2593$.

Hydrodynamic codes based on potential theory [20] are applied to compute the frequency-dependent added mass, potential damping and wave excitation forces. The data are then exploited to identify the memory function of main platform in pitch motion $K_{\theta_{r}}$ and the memory function of columns in heave motion $K_{c_{r}}$ using the toolbox developed by T. Perez and T. I. Fossen [21]. The results are shown in Fig. 5. It can be observed that the linear approximation models (shown in blue-dashed line) fit well with the computation (red line with circle markers) from potential theory. Characteristics of wave excitation forces on the pitch direction of main platform and on the heave direction of column, and their linear models are compared in Fig. 6. Owing to the non-causality and nonlinearity, the phases of the models are not coincident with the computation by potential theory. However, it is sufficient for our design purpose.

The proper ranges for $k$ and $c$ are set as $k \in[5,100]$ and $c \in[0.1,30]$ in this study, and the Particle Swarm Optimization algorithm provided by Matlab ${ }^{\circledR}$ is applied for solving the constrained optimization problem (28). The frequencies set for searching optimal values of $k$ and $c$ in (28) are linearly spaced in $\left[\frac{\omega_{0}}{2}, 3 \omega_{0}\right]$ with 100 points, which covers the wave frequency ranges shown in Fig. 4. The resulted optimal coefficients are

$$
\begin{aligned}
k_{\mathrm{opt}} & =5.00[\mathrm{~N} / \mathrm{m}], \\
c_{\mathrm{opt}} & =1.947[\mathrm{~N} \mathrm{~s} / \mathrm{m}] .
\end{aligned}
$$

From the results, it is observed that the optimal spring coeffi-
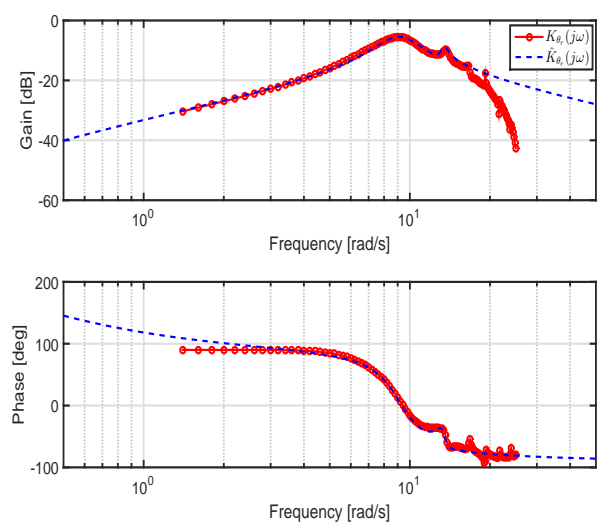

(a)

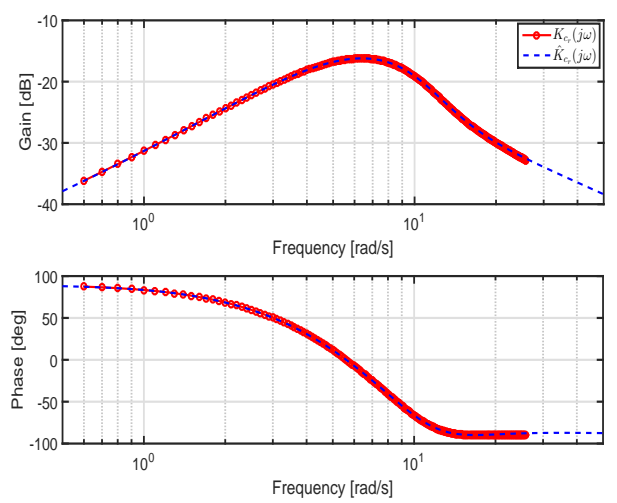

(b)

FIGURE 5. Memory functions computed from potential theory (red lines with circle markers) and their linear approximation (dashed lines).

cients hit the lower bound of the given range. This means the stronger the stiffness of spring is, the larger pitch motion would be excited. The perspective is also illustrated in Fig. 7(a) that the amplitude of pitch angle increases when $k$ varies toward the upper bound of the given range. Fig. 7(b) shows that the smallest pitching motion can be obtained by $c_{\text {opt }}$ when $c$ varies.

In addition, numerical results in time domain using wave exciting forces calculated from potential theory are shown in Fig. 9. The wave generated from (29) is shown in Fig. 8. It is also obtained that the calculated values from (28) can achieve smallest vibration. From these results we can also find that the model errors caused by linearizion in (16-18) do not affect the performance of the design. 


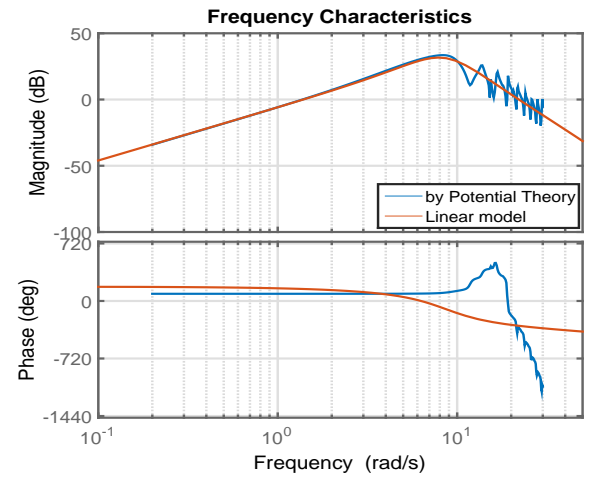

(a)

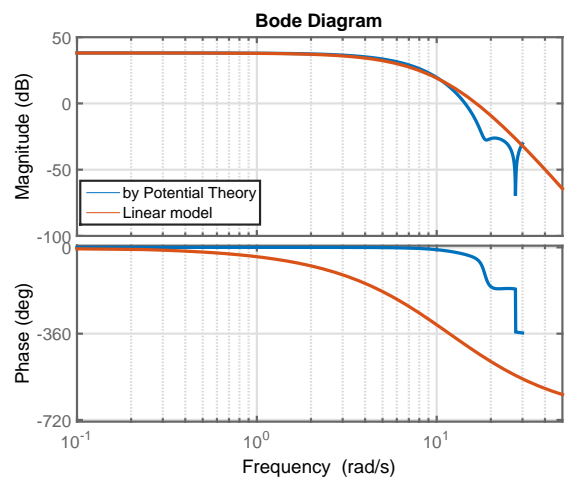

(b)

FIGURE 6. Wave excitation forces computed from potential theory (red lines with circle markers) and their linear approximation (dashed lines).

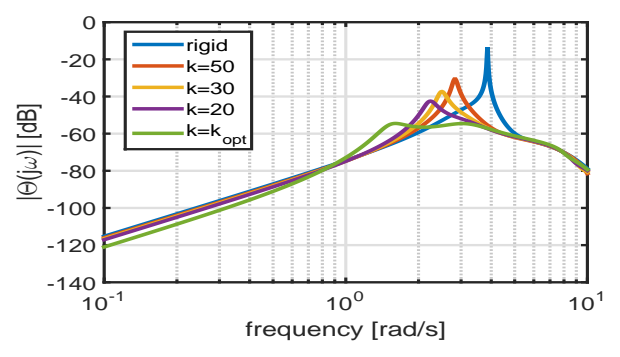

(a) Characteristics when $c=c_{\mathrm{opt}}$

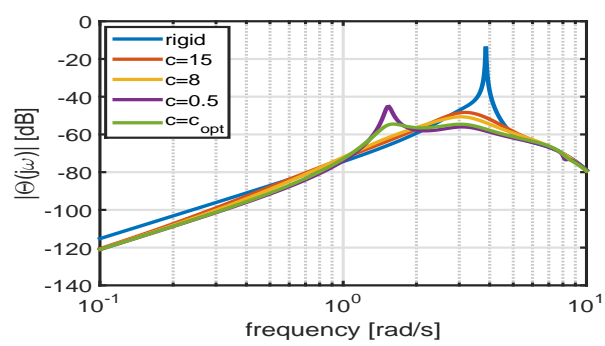

(b) Characteristics when $k=k_{\mathrm{opt}}$.

FIGURE 7. Frequency characteristics when varying $k$ and $c$ from the optimal values.

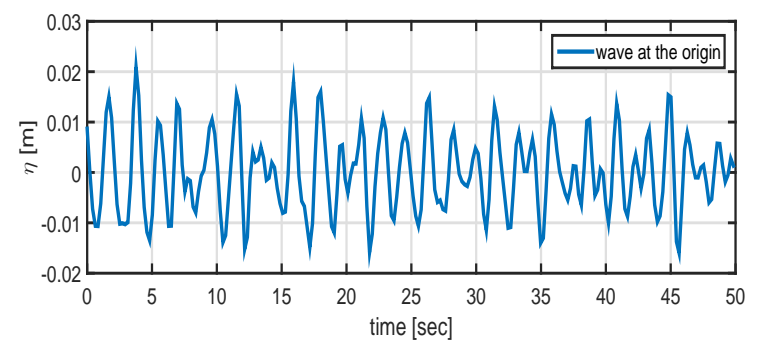

FIGURE 8. Wave at the origin based on (29).

\section{ACKNOWLEDGMENT}

This research was partially supported by JSPS KAKENHI Grant Number 15H06472.

\section{REFERENCES}

[1] Ishihara, T., Phuc, P., Sukegawa, H., Shimada, K., and Ohyama, T., 2007. "A study on the dynamic response of a semi-submersible floating offshore wind turbine system part 1: A water tank test". In Proceedings of the 12th International Conference on Wind Engineering, pp. 2511-2518.

[2] Henderson, A. R., and Patel, M. H., 2003. "On the mod- 


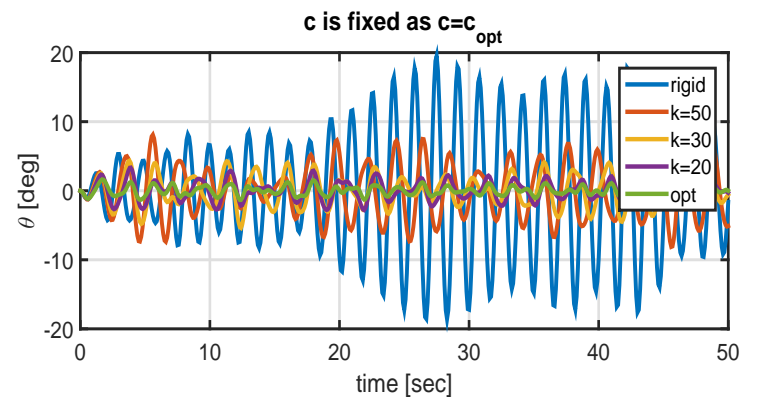

(a) Pitch motion when $c=c_{\text {opt }}$.

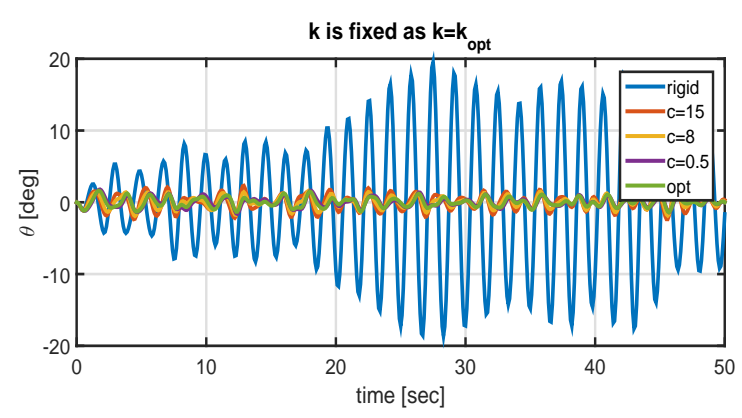

(b) Pitch motion when $k=k_{\text {opt }}$.

FIGURE 9. Numerical results when varying $k$ and $c$.

elling of a floating offshore wind turbine". Wind Energy, 6(1), pp. 53-86.

[3] Faltinsen, O., 1993. Sea Loads on Ships and Offshore Structures. Cambridge University Press, Cambridge.

[4] Sato, A., and Suzuki, H., 2006. "Effect of motion of floating platform on the strength design of floating wind turbine". In Proceedings of 28th Symposium on Wind Energy Utilization, pp. 192-195. in Japanese.

[5] Suzuki, H., and Sato, A., 2007. "Load on turbine blade induced by motion of floating platform and design requirement for the platform". In Proceedings of the 26th International Conference on Offshore Mechanics and Arctic Engineering, pp. 519-525.

[6] Huijs, F., Mikx, J., Savenije, F., and de Ridder, E.-J., 2013. "Integrated design of floater, mooring and control system for a semi-submersible floating wind turbine". In EWEA Offshore 2013 Vienna.

[7] Roddier, D., Cermelli, C., Aubault, A., and Weinstein, A., 2010. "Windfloat: A floating foundation for offshore wind turbine". Journal of Renewable and Sustainable Energy, 2, p. 033104.

[8] Karimirad, M., and Michailides, C., 2015. "V-shaped semisubmersible offshore wind turbine: An alternative concept for offshore wind technology". Renewable Energy, 83, pp. 126-143.

[9] Zhu, H., Ou, J., and Zhai, G., 2012. "Conceptual design of a deep draft semi-submersible platform with a movable heave-plate". Journal of Ocean University of China, 11(1), pp. 7-12.

[10] Aubault, A., Cermelli, C., and Roddier, D., 2006. "Structural design of a semi-submersible platform with waterentrapment plates based on a time-domain hydrodynamic algorithm coupled with finite-elements". In Proceedings of 6th International Offshore and Polar Engineering Conference, pp. 187-194.

[11] Roddier, D., and Cermelli, C., 2013. Column-stabilized offshore platform with water-entrapment plates and asymmetric mooring system for support of offshore wind turbines. patent, US008471396B2.

[12] Chandrasekaran, S., Raphel, D., and Shree, S., 2014. "Deep ocean wave energy systems (dowes): experimental investigations". Journal of Naval Architecture and Marine Engineering, 11(2), pp. 139-146.

[13] Perez, C., Greaves, D., and Iglesias, G., 2015. "A review of combined wave and offshore wind energy". Renewable and Sustainable Energy Reviews, 42, pp. 141-153.

[14] Perez, C., and Iglesias, G., 2012. "Integration of wave energy converters and offshore windmills". In Proceedings of the fourth international conference on ocean energy (ICOE).

[15] Cummins, W. E., 1962. The impulse response function and ship motions. Tech. Rep. 47, Schiffstechnik.

[16] Perez, T., and Fossen, T., 2009. Identification of dynamic models of marine structures from frequency-domain data enforcing model structure and parameter constraints. Tech. rep., ARC Centre of Excellence for Complex Dynamic Systems and Control.

[17] Yu, Z., and Falnes, J., 1995. "State-space modelling of a vertical cylinder in heave". Applied Ocean Research, 17, pp. 265-275.

[18] Fossen, T. I., 2011. Handbook of Marine Craft Hydrodynamics and Motion Control. John Wiley \& Sons, Ltd, UK.

[19] Kennedy, J., and Eberhart, R., 1995. "Particle swarm optimization". In Proc. IEEE Int. Conf. Neural Networks, Vol. 4, pp. 1942-1948.

[20] Liu, Y., Iwashita, H., and Hu, C., 2015. "A calculation method for finite depth free-surface green function". International Journal of Naval Architecture and Ocean Engineering, 7(2), pp. 375-389.

[21] Perez, T., and Fossen, T., 2009. "A matlab toolbox for parametric identification of radiation-force models of ships and offshore structures". Modeling, Identification and Control, 30(1), pp. 1-15. 